\title{
The effect of video game play on ophthalmic microsurgical simulator performance: a randomized controlled trial
}

\author{
Stephen C. Dryden, ${ }^{a}, *$ Ryan D. Gabbard, ${ }^{b}$ Fabliha Anbar, ${ }^{a}$ Shane D. Marsili, ${ }^{c}$ James C. Fleming ${ }^{a}$ and Brian T. Fowler ${ }^{a}$ \\ ${ }^{a}$ Department of Ophthalmology, Hamilton Eye Institute, University of Tennessee Health Science Center, Memphis, TN, USA; \\ ${ }^{b}$ Wright State University, Boonshoft School of Medicine, Dayton, OH, USA; ${ }^{c}$ Department of Ophthalmology, University of Kentucky \\ College of Medicine, Lexington, KY, USA \\ ${ }^{*}$ Corresponding author at: Department of Ophthalmology, Hamilton Eye Institute, University of Tennessee Health Science Center, \\ Memphis, TN, USA. Email: dryden.stephen.c@gmail.com
}

Date accepted for publication: 3 September 2021

\section{Abstract}

Objective: To examine the impact of real-time video game play on anterior segment ophthalmic microsurgical performance using a cataract surgical simulator (Eyesi). Methods: Medical students and ophthalmology residents at the University of Tennessee Health Science Center in Memphis, Tennessee, completed a questionnaire and baseline microsurgical evaluation using the capsulorhexis module on the surgical simulator. Participants were excluded if they had any previous intraocular surgical experience or extensive surgical simulator practice. Participants were randomized to group A (intervention) or group B (control). Group A completed as many levels of Tiltmasters as possible over a 30-min period, and group B was only allowed to watch the capsulorhexis demonstration video without any additional practice. After the 30-min time period elapsed, all participants performed the capsulorhexis module. Results: Of the 41 participants, regular video game players had greater baseline scores than non-players $(P=0.003)$ on the capsulorhexis module. There was no significant difference in the final surgical performance between the control and intervention groups. However, the intervention group showed significantly more improvement in surgical performance than the control group $(P=0.04)$. All participants demonstrated improvement in the final surgical performance regardless of whether they were in the control group or the intervention group. Conclusions: Regular video game play enhanced baseline microsurgical simulator performance. Both the control and intervention groups demonstrated improvement in final surgical simulator performance, indicating a warm-up effect. Participants in the intervention arm demonstrated significantly higher improvement than the control group, indicating skills transfer of the video game to surgical simulator performance after $30 \mathrm{~min}$ of intervention.

Keywords: video game; ophthalmic microsurgical simulator; anterior segment surgery; resident education; capsulorhexis; warm-up effect

\section{Introduction}

With the recent changes in resident work hour restrictions decreasing the amount of time for patient care, and increased emphasis on patient safety, there is a new, greater role for surgical simulation to improve patient care. Both laparoscopic and ophthalmic simulators have been shown to improve resident surgical performance without the need for traditional tissue-based wet lab models. ${ }^{1-4}$ Recent studies in the general surgery and endoscopic literature suggest a correlation between past and current video game use and improved laparoscopic and surgical performance. ${ }^{5-12}$ Chung et al. ${ }^{13}$ showed that gamers had higher baseline scores on an ophthalmologic surgical simulator than non-gamers, and Roohipor et al. ${ }^{14}$ showed that ophthalmic surgical simulator performance predicted subsequent surgical performance, but no video game-specific interventional studies specific to ophthalmology exist in the current literature. $^{13,14}$ The purpose of this study was to discern the influence of real-time video game play on ophthalmic microsurgical simulator performance.

\section{Methods}

Approval was obtained from the University of Tennessee Health Sciences Center Institutional Review Board. The study was compliant with the Health Insurance Portability 
and Accountability Act (HIPAA) and adhered to the tenets of the Declaration of Helsinki.

\section{Subject selection}

A single, blinded, randomized controlled trial was performed at the University of Tennessee Hamilton Eye Institute between May and July 2019 (Fig. 1). Volunteers were recruited from the School of Medicine and Ophthalmology Residency program. Those with any previous intraocular surgical experience or surgical simulator practice (greater than $1 \mathrm{~h}$ per week of residency training or previously completed the capsulorhexis module) were excluded. Participants then completed a short questionnaire related to demographics and past video game experience.

\section{Baseline evaluation of surgical performance}

Surgical simulator performance was assessed using the Eyesi surgical simulator (VR Magic, Mannheim, Germany) version V3.0.1. The pretesting phase was standardized for all participants. This included a demonstration video of the capsulorhexis module followed by orientation to the Eyesi surgical simulator led by the first author. The simulator provided instructions before the start of the task. Participants were then given $5 \mathrm{~min}$ to perform repetitions of the capsulorhexis module, a module that typically takes between 15 and $30 \mathrm{~s}$ to complete. Each outcome measurement as an Eyesi-generated score (minimum 0 points and maximum 100 points) was recorded and averaged as the baseline score.

\section{Study groups}

Participants were then randomly assigned to group A (intervention) and group B (control) using the GraphPad random number generator (GraphPad Software, San Diego, CA) after enrollment. Each participant was tested individually to prevent them knowing that there were two separate study groups.

\section{Video game intervention and control}

Tiltmasters (Dekovir Inc, Krasnoyarsk Territory, Russia) version V1.09.1 is a commercially available video game for download that is similar to mobile device balance games

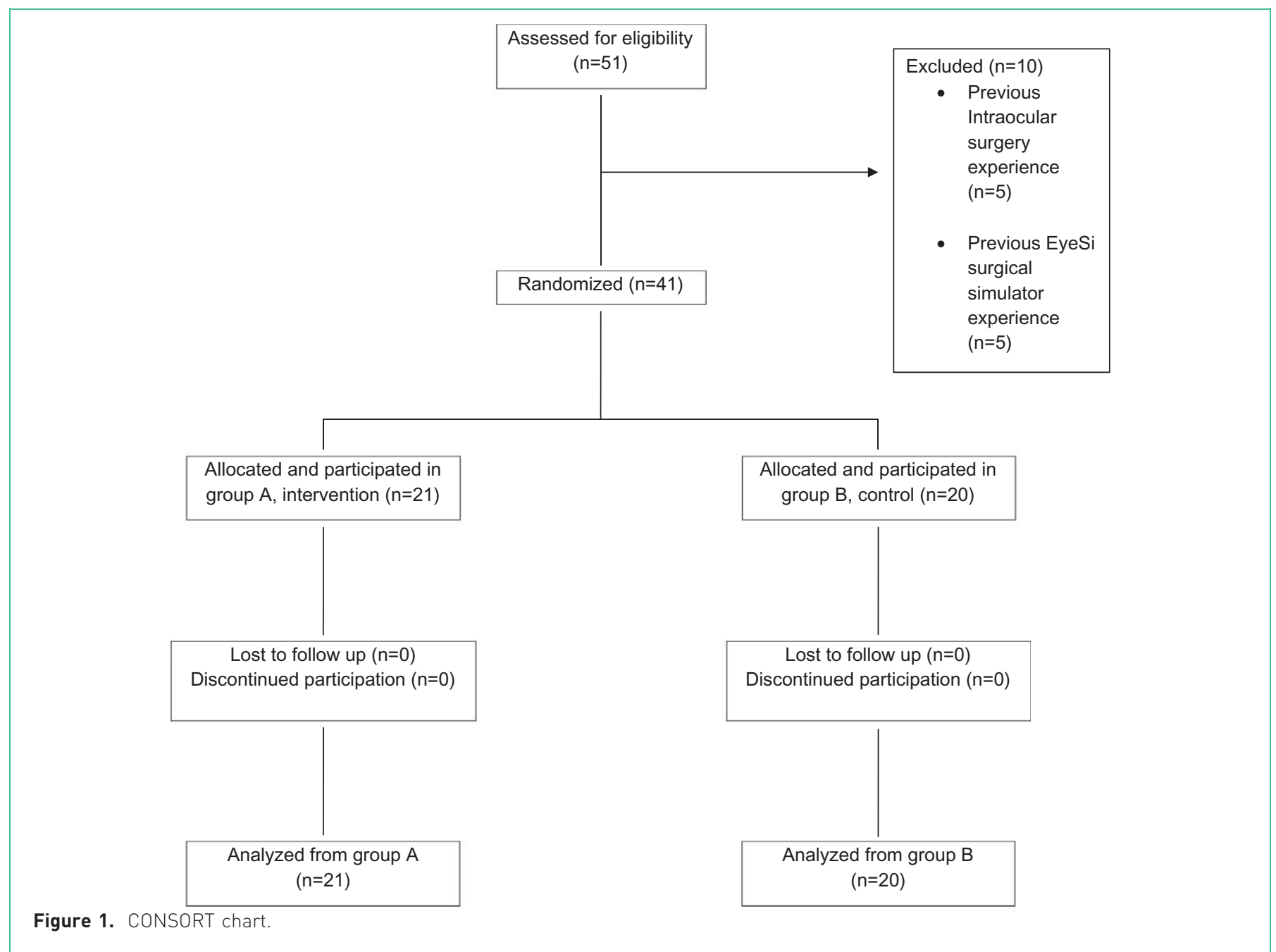


used in previous surgical simulation studies. ${ }^{9,11,15}$ The surgical application of this puzzle-based game involves the fine motor movements, three-dimensional depth perception, proprioception, and spatial distribution required to perform intraocular surgery. Participants assigned to group A were requested to complete as many levels of Tiltmasters as possible over a 30 -min time period. Participants in group B were allowed to watch the capsulorhexis demonstration video once without any other intervention or practice on the surgical simulator for a 30 -min time period.

\section{Final measurement of surgical performance}

After their 30-min time period, all participants were given $5 \mathrm{~min}$ to perform the capsulorhexis module. Each outcome measurement generated by the Eyesi was recorded and averaged as the final score.

\section{Statistical methods}

Statistical analyses were performed using SPSS software (IBM, New York, USA) from the University of Tennessee Health Science Center in Memphis, TN. Continuous variables were analyzed using a Mann-Whitney $U$ test and Kruskal-Wallis test with significance determined by $P<0.05$ due to the non-parametric nature of our dataset.

\section{Results}

\section{Demographics and surgical performance}

Table 1 presents the demographics, handedness, video game experience, and surgical simulator performance of the participants. The study included 41 participants aged 27.4 $( \pm 2.7)$ years. Twenty-three $(56 \%)$ were male and 18 (44\%) were female. Thirty-five $(85.3 \%)$ were right-handed and six (14.7\%) were left-handed. Seventeen (41.5\%) were third-year medical students, and 14 (34.1\%) were fourthyear medical students. Five (12.2\%) were ophthalmology interns (post-graduate year 1) and five were first-year ophthalmology residents (post-graduate year 2). Baseline surgical performance was higher in males than females $(P=0.002)$; however, final surgical performance was higher in females than males $(P=0.02)$. When adjusted for the control and intervention groups, there was no significant difference in surgical performance based on gender $(P=0.5)$. Right-handed participants had a lower mean baseline score $(63.4 \%$ versus $85.2 \%, P=0.4)$ and a higher mean score improvement $(13.2 \%$ versus $2.8 \%, P=0.04)$ compared with left-handed participants. Although there was no significant difference in baseline surgical performance based on training level, third-year medical students had the lowest mean baseline surgical performance $(P=0.2)$ and the most mean score improvement $(P=0.05)$.

Table 1. Demographics and video game experience

\begin{tabular}{|c|c|c|c|c|c|}
\hline \multirow[t]{2}{*}{ Variable } & & \multicolumn{2}{|c|}{ Baseline performance } & \multicolumn{2}{|c|}{ Score difference } \\
\hline & & Mean & $P$ value & Mean & $P$ value \\
\hline \multicolumn{6}{|l|}{ Gender, n (\%) } \\
\hline Male & $23(56)$ & 78.3 & & 6.6 & \\
\hline Female & $18(44)$ & 51.5 & & 18.2 & \\
\hline Age, years & $27.4 \pm 2.7$ & & $0.002^{*}$ & & $0.02^{*}$ \\
\hline Training level, n (\%) & & & $0.2^{* *}$ & & $0.05^{* *}$ \\
\hline MS3 & 17 & 53 & & 18.6 & \\
\hline MS4 & 14 & 78.1 & & 7.9 & \\
\hline PGY-1 & 5 & 70.8 & & 8 & \\
\hline PGY-2 & 5 & 79.2 & & 2.4 & \\
\hline Dexterity, n (\%) & & & $0.2^{*}$ & & $0.04^{*}$ \\
\hline Right & $35(85.3)$ & 63.4 & & 13.2 & \\
\hline Left & $6(14.7)$ & 85.2 & & 2.8 & \\
\hline Game experience, $\mathrm{n}(\%)$ & & & $0.003^{*}$ & & $0.04^{*}$ \\
\hline Yes & $25(61)$ & 77.8 & & 7.3 & \\
\hline No & $16(39)$ & 49 & & 18.8 & \\
\hline
\end{tabular}

$P$ values $<0.05$ are statistically significant. MS, medical student; PGY, post-graduate year.

*Mann-Whitney U test

*** Kruskal-Wallis test. 
Twenty-five (61\%) participants had video game experience and 16 (39\%) did not. Of those with video game experience, 14 were randomized to the intervention group (56\%) and 11 (44\%) to the control group. Participants with video game experience had a significantly higher baseline surgical score than those without experience $(P=0.003)$, and those without a history of video game experience had significantly higher interval improvement than those with experience $(P=0.04)$.

\section{Intergroup final surgical performance}

Table 2 presents the baseline surgical performance and final surgical performance based on the control versus intervention groups. There was a greater interval improvement in the intervention group than the control group (16.6 and 6.6, respectively, $P=0.04)$. There was no statistically significant difference in final surgical simulator performance based on the control and intervention groups (75.5 and 80.4 respectively, $P=0.6$ ).

\section{Discussion}

This study evaluated the influence of real-time video game play on anterior segment ophthalmic microsurgical simulator performance using the Eyesi surgical simulator. Participants with a history of video game play were found to have a significantly higher baseline surgical performance than those without. Although correlation does not imply causality, previous studies have shown that a history of video game play improves baseline microsurgical and laparoscopic surgical simulator performance. ${ }^{5-8,10,13,15,16}$ Even though the group with video game experience had a higher mean final score, the group without video game experience had significantly higher interval improvement than those with video game experience $(P=0.04)$. Given

Table 2. The baseline surgical performance and final surgical performance scores for the control and intervention groups

\begin{tabular}{lll}
\hline & Mean score & $P$ value $^{*}$ \\
\hline Baseline & & \\
Control & 69 & 0.9 \\
Intervention & 64.3 & \\
Final score & & 0.6 \\
$\quad$ Control & 75.5 & \\
Intervention & 80.4 & \\
Score difference & & 0.04 \\
Control & 6.6 & \\
Intervention & 16.6 & \\
\hline
\end{tabular}

$P$ values $<0.05$ are statistically significant.

* Mann-Whitney U test. the relatively equal distribution between the control and intervention groups, the difference in interval improvement may be attributed to the greater room for improvement due to lower baseline score in the group with no video game experience.

The randomized controlled trial portion of this study evaluated whether real-time video game play improved surgical simulator performance. Both the control and intervention groups showed improvement from the baseline to final surgical simulator performance, which may represent a "warmup" effect from the baseline capsulorhexis module as described by Rosser et al. ${ }^{8}$ Although there was no significant difference between the baseline score and the final surgical simulator score between the control and intervention groups, the intervention group had a significantly higher interval improvement than the control group. The duration of the intervention is important to note; there was a significant improvement after only $30 \mathrm{~min}$. The game type is also important. Tiltmasters was utilized for this study because it is a mobile device balance game similar to other balance games used by Plerhoples et al., ${ }^{9}$ Rosser et al., ${ }^{11}$ and Kazan et al. ${ }^{15}$ in their studies on video games and surgical skills training. In the future, this finding may be successfully applied not only to ophthalmology residency training programs but to any practicing anterior segment surgeon. A specialized ophthalmic-specific training game in the form of a mobile application can be developed as an adjunct or alternative personal surgical simulator. This game would provide a convenient, cost-effective, easily accessible training device available to residents and surgeons at any location and could also be used to warm up $30 \mathrm{~min}$ before surgery.

Limitations of this study include its power due to its relatively small sample size. The sample size was calculated based on the Yamane formula using a population size of 40 , indicative of the number of applicants our residency program interviews each academic cycle. Although our population was subject to selection bias (medical students and ophthalmology residents), we attempted to safeguard against skill discrepancy by recruiting only those without significant simulator experience (more than $1 \mathrm{~h}$ per week of residency training or previous capsulorhexis module experience) and only ophthalmology residents in their non-categorical internship and first categorical year in training at the beginning of the academic year, preventing any previous intraocular surgery experience. Despite the relatively small sample size and selection bias, this is the first randomized controlled trial of anterior segment ophthalmic surgical simulator training and real-time video game play to date.

In conclusion, participants with a history of previous video game play had higher baseline microsurgical performance 
on the anterior segment ophthalmic surgical simulator than those without. All participants showed improvement in the final surgical simulator performance, indicating a possible warm-up effect from baseline simulator assessment. However, participants randomized to the video game intervention arm had a significantly higher improvement in surgical performance than the control group, indicating a skills transfer from the video game to the surgical simulator after only $30 \mathrm{~min}$. Future studies can be performed to develop a specialized ophthalmology mobile application as an adjunct or alternative resource to site-specific surgical simulators for convenient, cost-effective personalized anterior segment ophthalmic surgical simulator training and warm up. Other models can be developed for application in other surgical specialties.

\section{Conflict of interests}

The authors have no competing interests to declare.

\section{Funding}

This research did not receive any specific grant from funding agencies in the public, commercial or not-for-profit sectors.

\section{References}

1. Crochet P, Netter A, Schmitt A, Garofalo A, Loundou A, Knight S, et al. Performance assessment for laparoscopic total hysterectomy in the operating room: validity evidence of a procedure-specific rating scale. J Minim Invasive Gynecol 2021. https://doi.org/10.1016/j.jmig.2021.02.013.

2. McCannel CA. Continuous curvilinear capsulorhexis training and non-rhexis related vitreous loss: the specificity of virtual reality simulator surgical training (an American Ophthalmological Society thesis). Trans Am Ophthalmol Soc. 2017; 115: T2.

3. Pokroy R, Du E, Alzaga A, Khodadadeh S, Steen D, Bachynski $\mathrm{B}$, et al. Impact of simulator training on resident cataract surgery. Graefes Arch Clin Exp Ophthalmol 2013; 251(3): 777-781. https://doi.org/10.1007/s00417-012-2160-z.

4. Solverson DJ, Mazzoli RA, Raymond WR, Nelson ML, Hansen EA, Torres MF, et al. Virtual reality simulation in acquiring and differentiating basic ophthalmic microsurgical skills. Simul Healthc 2009; 4(2): 98-103. https://doi.org/10. 1097/SIH.0b013e318195419e.

5. Harrington CM, Chaitanya V, Dicker P, Traynor O, Kavanagh DO. Playing to your skills: a randomised controlled trial evaluating a dedicated video game for minimally invasive surgery.
Surg Endosc 2018; 32(9): 3813-3821. https://doi.org/10. 1007/s00464-018-6107-2.

6. Sadandanan S, Dryfhout VL, Sosnowski JP. Video games and laparoscopic surgery. J Gynecol Surg 2008; 24(2): 67-74. https://doi.org/10.1089/gyn.2008.B-02295-1.

7. Sammut M, Sammut M, Andrejevic P. The benefits of being a video gamer in laparoscopic surgery. Int J Surg 2017; 45: 4246. https://doi.org/10.1016/j.ijsu.2017.07.072.

8. Rosser Jr JC, Gentile DA, Hanigan K, Danner OK. The effect of video game "warm-up" on performance of laparoscopic surgery tasks. JSLS 2012; 16(1): 3-9. https://doi.org/10. 4293/108680812X13291597715664.

9. Rosser Jr JC, Liu X, Jacobs C, Choi KM, Jalink MB, Ten Cate Hoedemaker HO. Impact of Super Monkey Ball and Underground video games on basic and advanced laparoscopic skill training. Surg Endosc 2017; 31(4): 1544-1549. https://doi.org/10.1007/s00464-016-5059-7.

10. Harbin AC, Nadhan KS, Mooney JH, Yu D, Kaplan J, McGinley-Hence N, et al. Prior video game utilization is associated with improved performance on a robotic skills simulator. J Robot Surg 2017; 11(3): 317-324. https://doi.org/10. 1007/s11701-016-0657-x.

11. Kazan R, Viezel-Mathieu A, Cyr S, Hemmerling TM, Lin SJ, Gilardino MS. Identification of new tools to predict surgical performance of novices using a plastic surgery simulator. J Surg Educ 2018; 75(6): 1650-1657. https://doi.org/10.1016/j. jsurg.2018.03.008.

12. Moglia A, Perrone V, Ferrari V, Morelli L, Boggi U, Ferrari $\mathrm{M}$, et al. Influence of videogames and musical instruments on performances at a simulator for robotic surgery. Minim Invasive Ther Allied Technol 2017; 26(3): 129-134. https://doi.org/10.1080/13645706.2016.1267018.

13. Chung AT, Lenci LT, Wang K, Collins TE, Griess MD, Oetting TA, et al. Effect of fine-motor-skill activities on surgical simulator performance. J Cataract Refract Surg 2017; 43(7): 915-922. https://doi.org/10.1016/j.jcrs.2017.05.024.

14. Roohipoor R, Yaseri M, Teymourpour A, Kloek C, Miller JB, Loewenstein JI. Early performance on an eye surgery simulator predicts subsequent resident surgical performance. J Surg Educ 2017; 74(6): 1105-1115. https://doi.org/10.1016/j.jsurg. 2017.04.002.

15. Plerhoples TA, Zak Y, Hernandez-Boussard T, Lau J. Another use of the mobile device: warm-up for laparoscopic surgery. J Surg Res 2011; 170(2): 185-188. https://doi.org/10.1016/j.jss. 2011.03.015.

16. Nomura T, Matsutani T, Hagiwara N, Fujita I, Nakamura Y, Kanazawa $\mathrm{Y}$, et al. Characteristics predicting laparoscopic skill in medical students: nine years' experience in a single center. Surg Endosc 2018; 32(1): 96-104. https://doi.org/10.1007/ s00464-017-5643-5. 